\title{
NUMERICAL ANALYSIS OF DEVELOPING TURBULENT FLOW IN A CLOSED COMPOUND CHANNEL
}

\author{
S. I. S. Souza ${ }^{a}$, \\ and J. N. V. Goulart ${ }^{\text {b }}$ \\ ${ }^{a}$ Laboratório de Mec. Computacional, LMC \\ Departamento de Engenharia Mecânica, UFRN \\ Campus Universitário, S/N - Lagoa Nova \\ CP: 59072-970, Natal, RN, Brasil \\ sandi@ufrnet.br \\ ${ }^{\mathrm{b}}$ Faculdade UnB Gama - UnB \\ Área Especial 2, Lote 14, Setor Central \\ CP: 72405-610 - Gama, DF, Brasil

\section{NOMENCLATURE}

$\begin{array}{ll}x, y, z & \text { space coordinates } \\ D & \text { width of test section, } \mathrm{m} \\ P & \text { depth of test section, } \mathrm{m} \\ L & \text { length of the test section, } \mathrm{m} \\ W & \text { width channel outlet, } \mathrm{m} \\ H & \text { height of channel, } \mathrm{m} \\ f & \text { frequency, Hz } \\ \mathrm{u}^{\prime}, \mathrm{v} & \text { components of velocity fluctuations, } \mathrm{m} / \mathrm{s} \\ D_{h} & \text { hydraulic-diameter, } \mathrm{m} \\ \mathrm{Re} & \text { Reynolds number } \\ N_{\text {seq }} & \text { average number of alternate sequence } \\ \mathrm{St} & \text { large vortices } \\ \mathrm{Be} & \text { Strouhal number } \\ \mathrm{y}^{+} & \text {bandwidth, Hz } \\ & \text { dimensionless coordinate from the wall, } \\ & \begin{array}{l}{ }^{*} \\ U_{\text {ent }}\end{array} \\ U_{c} & \text { entrance velocity, } \mathrm{m} / \mathrm{s} \\ & \text { convection velocity, } \mathrm{m} / \mathrm{s}\end{array}$

\begin{abstract}
The study of turbulence characteristics in compound channels is still focus of attention. A lot of experimental results have been produced. Main results have revealed a mixing layer formation between main subchannel and the gap region, implying the flow might be ruled by local scales.

The outcomes have pointed to the instabilities of mixing layer are responsible for large structures formation between main channel and narrow gap. Furthermore, the periodical behavior of these structures seems to be ruled by mean mixing layer characteristics, as velocity difference, velocity of convection and mixing layer thickness. By using ANSYS-CFX-12, with unsteady Reynolds Average Navier-Stokes and as turbulence model SpalartAllmaras (SA), a compound channel was studied. Numerical results predicted velocity profile with high vorticity peaks and flow instabilities starting at $L / D_{h}=15$.
\end{abstract}

Keywords: turbulence in compound channels,flow pulsations, numerical simulation, Spalart-Almaras modeling

\section{Greek symbols}

$\begin{array}{ll}\rho & \text { density, } \mathrm{kg} / \mathrm{m}^{3} \\ \delta_{(x)} & \text { mixing layer thickness, } \mathrm{m} \\ \lambda & \text { wavelength, } \mathrm{m} \\ \Phi(u u) & \text { autospectral density function, } \frac{\mathrm{m}^{2}}{\mathrm{~s}^{2}} \mathrm{~Hz}^{-1} \\ \Phi(v v) & \text { autospectral density function, } \frac{\mathrm{m}^{2}}{\mathrm{~s}^{2}} \mathrm{~Hz}^{-1}\end{array}$

\section{INTRODUCTION}

Compound channels are characterized by the presence of a narrow region connecting one or more main channels, Figure 1. The narrow gaps are responsible for mass, energy and momentum exchanging between main subchannels.
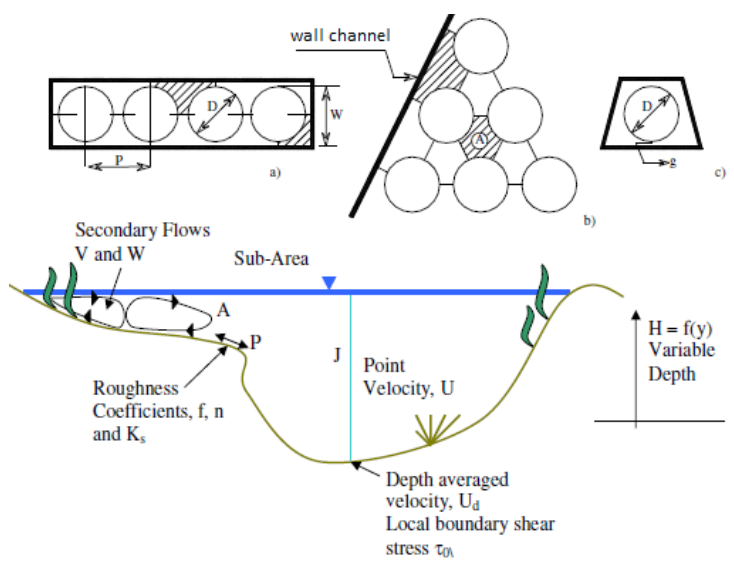

Figure 1. Examples of compound channels.

These geometries are present in many areas of the engineering, as nuclear reactors, steam generators and some kinds of water channels. On the mechanical engineering field the problems are often linked to heat exchangers, nuclear reactor fuel elements and even electronic devices. On the last ones, the main goal is raising heat exchange rates. A comprehensive review of the flow in compound channels was presented by Meyer (2010).

As remarkable features of these kinds of channels are the unusual Reynolds stresses distribution and the sinusoidal patterns of the velocities series taken from these channels, as well. 
Being these facts very well reported by Knight and Shiono (1990), Soldini et al. (2004), when the authors studied open compound channels. Related to closed compound channels the works from Meyer and Rehme (1994 and 1995), showed similar results those previously found in open channels.

Despite unusual Reynolds stress distribution, the main source of interest has lain on the unexpected sinusoidal patterns of velocity fluctuations near the gap. The quasi-periodical behavior of the velocity fluctuations was named flow pulsations.

Flow pulsations occurrence was reported, at the first time, by Rowe (1974), when the author studied mixture process of the turbulent flow in a rod bundle. According to author, the frequency associated to this phenomenon increased when the gap width was reduced. By using hot wires probes Möller (1991), confirmed Rowe's findings, therefore, his results also showed flow pulsations were associated to strong vorticity field near the gap.

Quasi-periodical flow pulsations were also found in other kind of compound channel, showing this phenomenon was not restricted to rod bundles assembly. $\mathrm{Wu}$ and Trupp (1994) performed hot wire measurements in a trapezoidal channel containing a single tube. The results showed pronounced peaks in spectra, confirming the strong dependence of the frequency on geometrical parameters and the flow velocity. After that, Meyer and Rehme (1994, 1995) performed measurements in unusual compound channels. By using hot wire anemometry, the authors studied the flow characteristics in a channel with two or several parallel fins attached to a side wall. The geometry was quite similar to an internally finned channel, forming slots connected to main channels. Test sections were characterized by dimensionless parameter, $p / d$, being $p$ the depth of the slot formed by the plates and $d$ the distance between two plates. Test sections with $p / d$ values ranging from 1.66 to 10.0 were investigated. Regarding mean turbulent quantities, all features stressed in the previous papers were confirmed. When attention was pointed to large scale structures, the authors observed flow pulsations for $p / d \geq 2$, confirming results by using flow visualizations. A correlation for the Strouhal number was also proposed. The Strouhal number definition was based on the main frequency, the square root of the product of $p$ and $d$ and the edge velocity, measured at edge of plates, $U_{e d}$. Nevertheless, the results showed discrepancies for $p / d$ values greater than 7.

One of the 3 compound channels studied by Meyer and Rehme (1995), was also investigated by Goulart and Möller (2006, 2007). In the first work (Goulart and Möller, 2006), the authors investigated a rectangular channel with two parallel plates attached on the lateral wall. By using hot wires were performed measurements of two components of velocities for six test sections. For test sections depth- width ratio remained constant $p / d=5$, even though dimensional parameters, $p, d$, could be different.

Despite the findings reported by Meyer and Rehme (1995), large-scale structures could not be found in all test sections, but only in that one with the smallest width, $d$.

The conclusions led to a second investigation that showed the strong relationship between axial velocities profiles and the presence of large-scales structures. In Goulart and Möller (2007), the authors were succeeded in obtaining comprehensive measurements of the axial and transversal velocities fluctuations in ten test sections, involving three $p / d$ ratios, 5, 10 and 12.50 . The results showed a steady state plane turbulent mixing layer in spatial development between the plates. By using selfsimilarity functions it was possible to describe mean axial velocity (at the symmetry lines) as a tangent hyperbolic function. Related to flow large-scales appearances, an attempt to make a correlation for Strouhal number was done. The Strouhal number was defined as that one showed in mixing layer problems, using mixing layer thickness, $\delta_{(x)}$, as length scale, and the convection velocity, $U_{c}$, as velocity scale. The Strouhal numbers remained constant even for the deepest test section, where $p / d=12.50$.

By employing a commercial code CFX-12 and Spalart-Allmaras as turbulence model, turbulent flow in as compound channel was simulated. Simulations were based on experimental data from Goulart (2009), in a $L / D_{h}=10$, compound channel. Results also could be compared with those available in Home et al. (2009), who employed the same numerical facilities. Large scale structures appearance (flow pulsations) were also the target of this paper. In order to attain this goal the initial domain was made longer than experimental model. Large scales could be seen from $L / D_{h}=15$.

\section{COMPUTATIONAL DOMAIN}

The test section, Figure 2, consists in a 2600 $\mathrm{mm}$ long channel where both the width, $W=50 \mathrm{~mm}$ and height, $H=54 \mathrm{~mm}$. A slot with dimensions depth, $P=25 \mathrm{~mm}$ and width, $D=4 \mathrm{~mm}$, was attached to main channel connect then by a gap. To perform numerical simulation the working fluid was air at 25 ${ }^{\circ} \mathrm{C}$, reaching the test section with about $5 \%$ turbulence intensity. No slip was imposed for walls. Mass flux was applied at the entrance region, yielding a Reynolds number, $\operatorname{Re}=84.34 \times 10^{3}$. Reynolds number was defined using mean velocity at the entrance, $U_{\text {ent }}=25,14 \mathrm{~m} / \mathrm{s}$, kinematic viscosity, $v$, and hydraulic diameter of the channel, $D_{h}$.

Figure 2, also shows details about coordinate axis and mesh. Mesh build was done by using ANSYS-ICEM, and the first grid point from walls was based on dimensionless coordinate, $y+=1$. This constrains yielded 4386200 nodes. Simulations were 
run in a workstation Dell T7400, taking approximately 10 days.

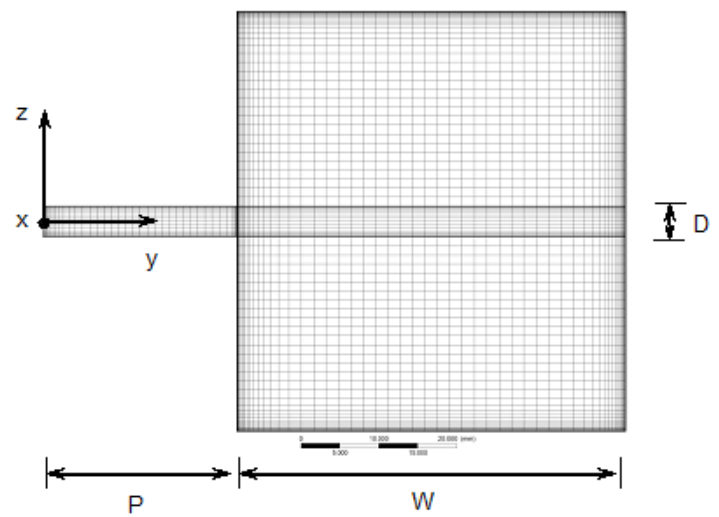

Figure 2. Computational domain.

In the simulation, the convergence criteria and the time step were specified as $1.50 \times 10^{-6}$, for r.m.s value, and $10^{-4} \mathrm{~s}$, respectively. Simulation took time enough to attain a distance $L=60 D_{h}$.

\section{GOVERNING EQUATIONS}

\section{Mass and Momentum Conservation}

For incompressible flow mass equation and Navier-Stokes for URANS can be written as:

$$
\begin{gathered}
\frac{\partial \overline{u_{i}}}{\partial x_{i}}=0 \\
\frac{\partial \overline{u_{i}}}{\partial t}+\frac{\partial\left(\overline{u_{i} u_{j}}\right)}{\partial x_{j}}=-\frac{1}{\rho} \frac{\partial \bar{p}}{\partial x_{i}}+\ldots \\
\ldots+v \frac{\partial^{2} \overline{u_{i}}}{\partial x_{j}^{2}}-\frac{\partial \tau_{i j}}{\partial x_{j}}
\end{gathered}
$$

The over bar symbols in Eq. (1) and (2), represents mean quantities and $\tau_{i j}$, represents the Reynolds stress tensor.

$$
\tau_{i j}=-\rho \overline{u_{i} u_{j}}
$$

By using eddy viscosity concept non-linear terms of velocity fluctuation can be modeled as follow:

$$
-\overline{u_{i} u_{j}}=v_{t}\left(\frac{\partial \overline{u_{i}}}{\partial x_{j}}+\frac{\partial \overline{u_{j}}}{\partial x_{i}}\right)-\frac{2}{3} \delta_{i j} k
$$

where $v_{t}$, is eddy viscosity and is obtained from specific RANS model, overcoming the closure problem.
Spalart-Allmaras model, Spalart and Allmaras (1992), is a one model equation, involving a transport equation for modified viscosity, $\tilde{v}$.

$$
\begin{gathered}
\frac{\partial \rho \tilde{v}}{\partial t}+\frac{\partial\left(\rho \overline{u_{i}} \tilde{v}\right)}{\partial x_{i}}=\frac{1}{\sigma_{\tilde{v}}}\left\{\frac{\partial}{\partial x_{j}}\left[(\mu+\rho \tilde{v}) \frac{\partial \tilde{v}}{\partial x_{j}}\right]+\ldots\right. \\
\left.\ldots+C_{b 2} \rho\left(\frac{\partial \tilde{v}}{\partial x_{j}}\right)^{2}\right\}+G_{v}-Y_{v}
\end{gathered}
$$

Now the eddy viscosity, $v_{t}$, can be computed from

$$
\begin{aligned}
& v_{t}=\tilde{v} f_{v 1} \\
& f_{v 1}=\frac{\chi^{3}}{\chi^{3}+C_{v 1}^{3}} \\
& \chi \equiv \frac{\tilde{v}}{v}
\end{aligned}
$$

where, $f_{v 1}$, is the viscous damping function, $C_{b 2}$, is $0.622, \underset{v}{\sigma_{\sim}}=2 / 3$ and $C_{v l}$, is 7.10 .

In the production and destruction terms, $\tilde{S}$ and $d$, represents, respectively, modified velocity gradient and distance from the wall. The term, $C_{b I}$ is constants being 0.1355 .

$$
\begin{aligned}
& \tilde{S}=S+\frac{v}{\kappa^{2} d^{2}} f_{v 2} \\
& f_{v 2}=1-\frac{\chi}{1+\chi f_{v 1}}
\end{aligned}
$$

Other functions are stressed in Eq. (7).

Further details should be seen in SparlatAllmaras (1992).

\section{RESULTS AND DISCUSSION}

\section{Velocity Profiles}

Figure 3 (a), shows mean axial velocity distribution at different stations along the channel and the experimental data as well.

Numerical and experimental results are stressed in dimensionless form by using its maximum values. One can see mean velocities profiles were almost unchangeable from $1000 \mathrm{~mm}$ after entrance. Inspite of the fact the experimental and numerical predictions had been taken at different stations, it is important to note main features stressed in Figure 3(a) and 3 (b), are still present. 


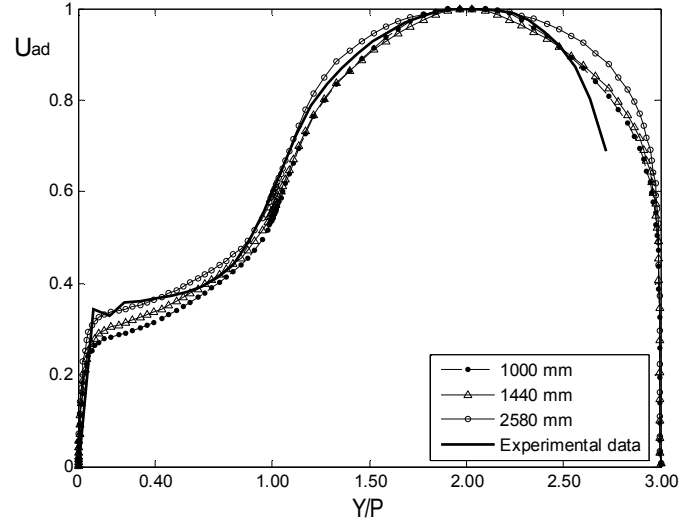

a)

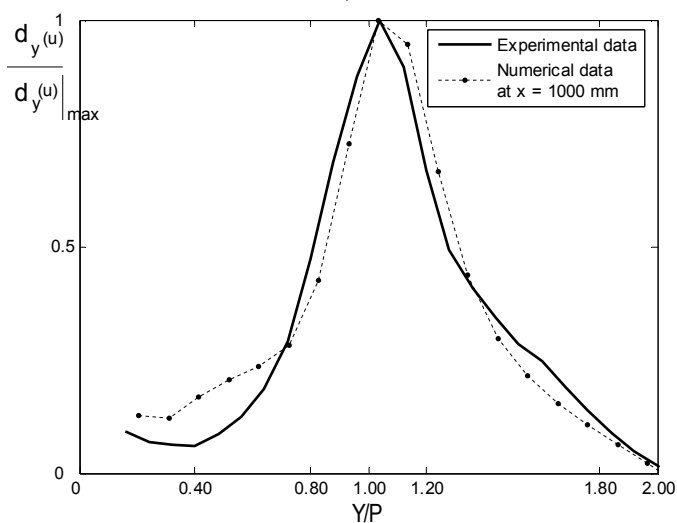

b)

Figure 3. Axial velocity distribution and its derivative form. (a) mean axial velocity profile. (b)

Experimental and numerical results for derivative form of the velocity profile. Experimental data from Goulart (2009).

Different authors have reported similar behavior that one found here. Flow acceleration towards the main channel followed by a velocity reduction in the narrow gaps and a turning point in the mean velocity profile, Figure 3(b).

Flow acceleration can be seen in Figure 4 (a), similar results are also found by Home et al. (2009), when the authors studied twin compound channel, Figure 4 (b). Both results are quite similar to Meyer and Rheme's findings (Meyer and Rehme, 1994), when the authors carried out studies in twin compound channels.

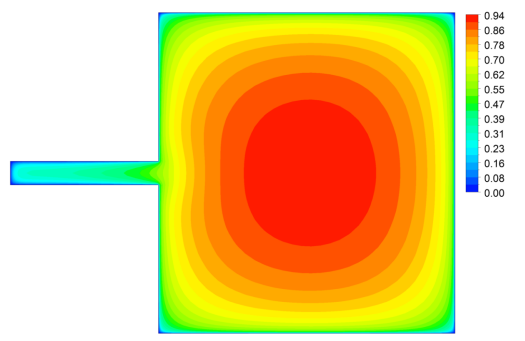

a)

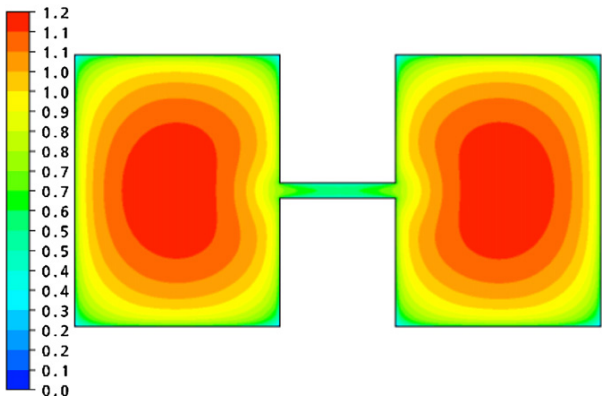

b)

Figure 4. Axial velocity distribution. (a) 2-D - plot of mean axial velocity field - numerical data. (b) 2-D plot of mean axial velocity field from Home et al.(2009).

\section{COHERENT STRUCTURES}

Time traces axial and spanwise velocities fluctuations are depicted in Figure 5 (a) and (b). All velocity data are result from numerical analysis and taken at slot/main subchannel interface, $Y / P=1$. Numerical data were computed at different stations along the channel's length, $500 \mathrm{~mm}$ and $2580 \mathrm{~mm}$, after channel's entrance, respectively.
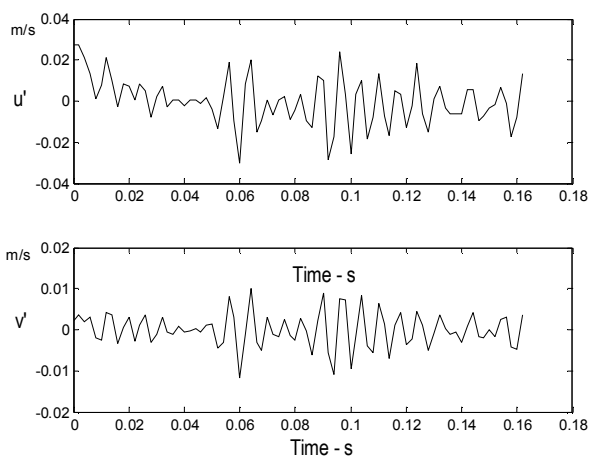

a)
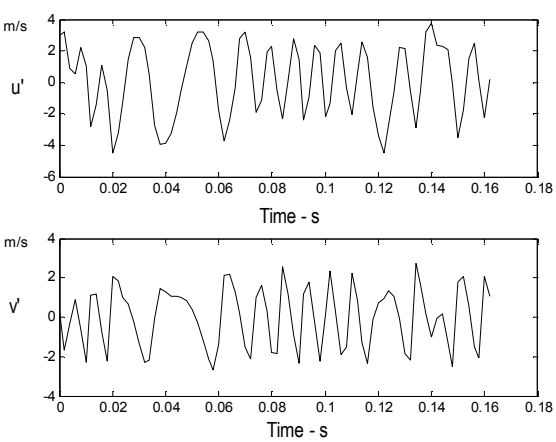

b)

Figure 5. Time traces of axial and spanwise velocity fluctuations. (a) $500 \mathrm{~mm}$ from channel's entrance. (b) $2580 \mathrm{~mm}$ from channel's entrance. 
Velocities time-record from Figure 5 (a) were taken at $L / D_{h}=10$, whereas, data from Figure 5 (b) were taken at $L / D_{h}=49$. From both figures one can see remarkable difference between then. Starting with velocity fluctuation peaks. When compared velocity data from $L / D_{h}=49$, axial and span-wise component values are quite similar, implying in similar r.m.s value. It is also important to note velocities time records from Figure 5 (b), present quasi-periodical behavior suggesting large coherent structures in the flow at this position. However, when attention is turned to velocity data from $L / D_{h}=10$, the same is no true. Velocity peaks are lower than those found downstream and quasi-periodical behavior could not be detected.

Another noteworthy feature in problems involving compound channels concerns the streamwise vortex formation. Coherent structures, in these sorts of flows, have been well known for at least two decades. Knight and Shiono's findings, from Shiono and Knight (1990), have reported these structures appearance since then. The identification and their characterization have been done for several purposes. First, from the energetic view point, according to Brownd and Ho (1983), more than 50\% of the turbulent kinetic energy is attributable to the large-vortices. Secondly, because the dynamical properties, coherent structures play an essential role in mixing processes, drag, noise emission, heat transfer, and others diffusive processes.

Investigations on coherent structures in the flow were also performed for test section. Figure 6 (a) and (b), shows instantaneous spanwise velocity component along the channel, by using a slice plane at the middle height of the channel. There, one can see flow instabilities starting, roughly, at $L / D_{h}=15$. Stable vortices rotating in opposite directions appear inside the slot, keeping a constant spacing between then travelling a certain velocity, named convection velocity, $U_{c}$.
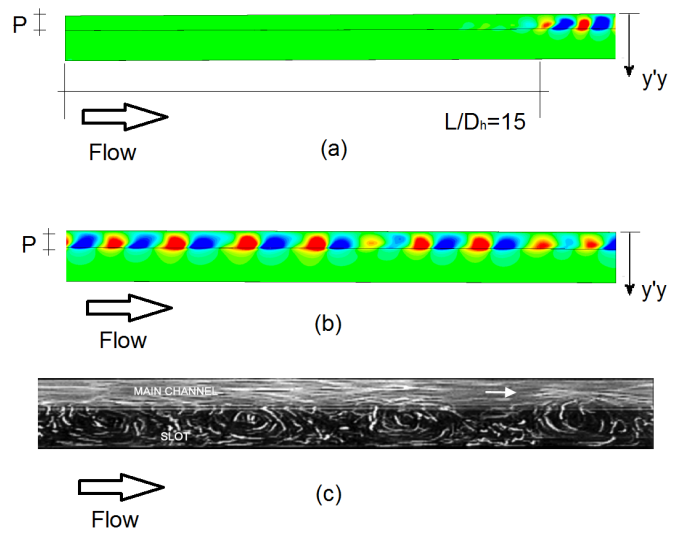

Figure 6. Instantaneous spanwise velocity contours predicted by Spalart-Allmaras turbulence model. (c)

Flow visualization of eddies moving in the

longitudinal slot - Meyer and Rheme (1995).
Large-scale structures dominating the slot, travelling at constant velocity and rotating in opposite directions were predicted by using LES-calculations in Biemüller et al. (1995) and Home et al. (2009). Figure 6 (c), shows flow visualization in a water channel, performed by Meyer and Rheme (1995). Flow visualization was carried out in a quite similar compound channel to that used in the present work, with $P / D=5.78, \operatorname{Re}=2.00 \times 10^{5}$ and $L / D_{h}=52.23$.

It is possible to determine velocity transportation of the vortex as follow

$$
U_{c}=\lambda f
$$

where, the wavelength, $\lambda$, is the streamwise spacing between alternate sequence of large structures and $f$, is the main frequency.

The wavelength associated with alternate sequence of vortex can be estimated by using the average number of these structures in a certain domain, Home et al. (2009). Taking a picture of these structures and knowing the length of domain, for example Figure 6 (b), it is possible estimate $\lambda$ by using

$$
\lambda=\frac{L}{N_{\text {seq }}}
$$

where, the length of domain is $L$ and $N_{\text {seq }}$, is the average number of alternate sequence of structures. For this problem Eq. (9), yields, $\lambda=110 \mathrm{~mm}$. To estimate the convection velocity, $U_{c}$, is necessary to know main frequency on the time trace velocities record. However, due hardware limitations a long time-serie for velocity fluctuation was not accomplished. Transient data of velocity were taken for only $0.162 \mathrm{~s}$.

Figure 7, shows autospectral density functions for velocities series from Figure 5 (a) and (b). There, one can see peaks in the spectra for both velocities components located at $2580 \mathrm{~mm}, L / D_{h}=49$. On the other hand, velocity series taken at $500 \mathrm{~mm}, L / D_{h}=$ 10 , did not present any peak in the spectra. In addition, energy of velocity fluctuations taken at $L / D_{h}$ $=10$, is lower than that one taken at $L / D_{h}=49$.

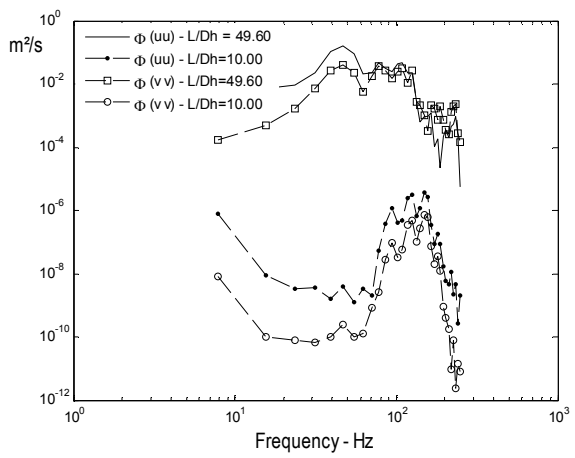

Figure 7. Autospectral analisis of numerical data of velocity fluctuations. 
Despite width band peak in the spectra, located roughly at $50 \mathrm{~Hz}$, is possible to see the frequency of the structures. Actually, a carefully examination showed this frequency as $46.50 \mathrm{~Hz}$. This number is less than half that expected. Works from Goulart and Möller (2008) and Goulart (2009), showed Strouhal number could be formulated by using average scales from mean axial velocity profiles.

$$
S t=\frac{f \delta_{(x)}}{U_{c}}
$$

In Eq. (10) the scales named, $f, \delta_{(\mathrm{x})}$, and $U_{c}$, are the frequency of the structures, the mixing layer thickness, and the velocity convection. By performing experimental measurements and based on the results, the authors suggested a mixing layer formation between main subchannel and narrow gaps. This fact may give rise to large-scale structures like similar to Kelvin-Helmholtz instabilities. For this kind of flow Strouhal numbers collapsed on 0.10.

Following, Eq. (10), and the results from previous works, the expected frequency is $115 \mathrm{~Hz}$. The present simulation under predicted the frequency of the vortex by a factor higher than 2 . However, it was not a completely unexpected result. Home's simulation (Home et al., 2009), using the same turbulence model had their frequency results over predicted by a factor of 1.825 .

After taking the frequency, $f$, and the wavelength, $\lambda$, associated with these large structures, the Eq. (8), yields the convection velocity, $U_{c}=5.11$ $\mathrm{m} / \mathrm{s}$. By follow experimental evidences in Meyer and Rheme's work (Meyer and Rehme, 1995), it is observed convection velocity was under predicted by numerical simulation. According to the authors the edge velocity, $U_{e}$, and convection velocity, $U_{c}$, ratio, should be within 1.37. In present work $U_{e} / U_{c}$, ratio was 2.73 .

\section{CONCLUSIONS}

In this paper turbulent flow in a compound channel was analyzed. By using a commercial code, CFX-12, and Spalart-Almaras, as turbulence model, the flow behavior could be predicted. Results could be compared with experimental data from Goulart (2009) and numerical data from Home et al. (2009).

The predicted velocity profile showed the presence of high vorticity peak at the interface between main channel and narrow gap. Low velocity could be found in the narrow gap and higher one in the main channel. These results match those ones found previously in Meyer's papers (Meyer e Rheme, 1994 and 1995), Home et al. (2009) and Goulart (2009). It is also a noteworthy feature the presence of the turning point, necessary condition for instabilities, those could be predicted by the code.
Making the channel longer than original, timeseries of velocity fluctuations revealed periodical pattern that started only at $L / D_{h}=15$. Although, the code had been able to predict large-scales structures dominating the slot and travelling at constant velocity, it is not possible to match the expected frequency. The frequency of these structures was less than half that it should be.

Despite over prediction the frequency the turbulence model was able to show the large vortex, rising awareness about how to deal with this sort of flow in the next simulations. Although, had not been mentioned, SST turbulence model, with unsteady Reynolds Average Navier-Stokes was also employed. Neither flow pulsations nor good results for velocities profiles could be found.

\section{REFERENCES}

Biemüler, M., Meyer, L., and Rehme, K., 1996, Large Eddy Simulation and Measurement of the Structure of Turbulence in Two Rectangular Channels Connected by the Gap, in: 3rd International Engineering Turbulence Modeling and Experiments, Heraklion, GR, pp. 249-258.

Browand, F. K., and Ho, Chih-Ming., 1983, The mixing layer: An example of quasi twodimimensional turbulence, Journal de Mécanique théorique et appliquée, Numéro spécial, pp. 99-120.

Goulart, J. N. V., and Möller, S. V., 2006, Flow pulsations in short compound channels, in: $11^{\text {th }}$ Brazilian Congress of Thermal Science and Engineering, Curitiba-PR, Brazil.

Goulart, J. N. V., and Möller, S. V., 2007, Shear Flow in Compound Channels, in: 19th International Congress of Mechanical Engineering, Brasília, Brazil.

Goulart, J. N. V., and Möller, S. V., 2008, Turbulent Flow in Closed Compound Channels, in: 12th Brazilian Congress of Thermal Science and Engineering, Belo Horizonte, Brazil.

Goulart, J. N. V., 2009, Escoamentos Cisalhantes em Canais Compostos, Doctoral Thesis, Universidade Federal do Rio Grande do Sul, Porto Alegre, RS.

Home, D., Arvanitis, G., Linghtstone, M. F. and Hamed, M. S.,2009, Simulation of Flow Pulsation in a Twin Rectangular Subchannel Geometry Using Unsteady Reynolds average Navier-Stokes modelling, Nuclear Engineering and Design,Vol. 239, pp. 2964-2980.

Knight, D.W., and Shiono, K., 1990, Turbulence Measurements in a Shear-Layer Region of a Compound Channel, Journal of Hydraulic Research, Vol. 28, pp. 175-196.

Meyer, L., and Rehme, K., 1994, Large-scale turbulence phenomena in compound rectangular channels, Experimental Thermal and Fluid Science, Vol. 8, pp. 286-304. 
Meyer, L., and Rehme, K., 1995, Periodic vortices in flow though channels with longitudinal slots or fins, in: 10th Symposium on Turbulent Shear Flows, University Park, Pennsylvania, pp. 249-258.

Meyer L., 2010, From Discovery to Recognition of Periodic Large Scale Vortices in Rod Bundles as Source of Natural Mixing Between Subchannels-A review, Nuclear Engineering and Design, Vol. 240, pp. $1575-1588$.

Möller, S. V., 1991, On Phenomena of Turbulent Flow through Rod Bundles, Experimental Thermal and Fluid Science, Vol. 4, pp. 25-35.

Rowe, D.S., Johnson, B.M., and Knudsen, J. G., 1974, Implications Concerning rod Bundle Crossflow Mixing Based on Measurements of Turbulent Flow Structure, International Journal of Heat and Mass Transfer, Vol. 17, pp. 407-419.

Soldini, L., Piattella, A., Brocchini, M., Mancinelli, A. and Bernetti, R., 2004, MacrovorticesInduced Horizontal Mixing in Compound Channels, Ocean Dynamics, Vol. 54, pp. 333-339.

Spalart, P. R., and Allmaras, S.R., 1992, A oneequation turbulence model for aerodynamic flow, in: 30th Aerospace Science Meeting and Exhibit, Nevada, pp. 92-0439.

Received: July 30, 2011

Revised: August 30, 2011

Accepted: September 30, 2011 